\title{
La tradition conjuguée au présent Une ethnographie du spectacle AfroBrasil
}

\author{
Emília Chamone
}

\section{Introduction}

Diffusée initialement par des immigrés brésiliens installés en Île-de-France à partir du milieu des années 1970, une certaine musique percussive brésilienne a entraîné une multiplicité de pratiques culturelles et une intense production musicale sous le signe des " batucadas ". Mot-valise qui recouvre une grande gamme de pratiques et de musiques, la batucada peut être définie actuellement comme un groupe de percussions « d'inspiration brésilienne ${ }^{1}$ ». Trois pratiques musicales brésiliennes constituent ses principales sources d'inspiration : la samba-reggae, l'école de samba et le maracatu. Ces groupes de percussions français consacrés à la musique brésilienne s'approprient un répertoire de rythmes carnavalesques avec beaucoup de liberté, donnant naissance à des pratiques qui peuvent être très éloignées de celles dites « inspiratrices ", mais qui, dans cet écart, fabriquent différents horizons d'attente et de nouvelles sonorités. De nouveaux espaces de convivialité et de sociabilité sont établis autour de ces groupes qui rassemblent des musiciens amateurs et professionnels, des francophones d'origines différentes.

Visibles et audibles dans la formation instrumentale de chaque ensemble, dans son répertoire de rythmes, de costumes et de chorégraphies, les éléments perçus comme « brésiliens " à l'intérieur des batucadas sont également visibles à travers une gestualité spécifique et une façon propre

\footnotetext{
1 Voir la revue de presse du Concours de batucadas de Paris, http://www.noitesdobrasil.com. Cette définition, assez large et opératoire, a plusieurs avantages comme celui de regrouper dans une seule catégorie tous les groupes qui interprètent un répertoire considéré comme brésilien ou qui utilisent des instruments de percussions identifiés comme brésiliens.
} 
d'interpréter et de composer la musique. Pourtant, de cette profusion de rythmes, de gestes et d'instruments qui composent le répertoire musical des batucadas, se dégagent plusieurs points communs qui touchent toujours au caractère collectif, festif et déambulatoire de ces pratiques. Ainsi, bien que l'on puisse remarquer qu'au niveau purement musical les batucadas sont très hétérogènes, elles s'accordent à la manière collective de faire de la musique percussive et au partage d'un certain imaginaire brésilien.

Dans cet article je propose d'aborder la fabrication d'une musique brésilienne à Paris en suivant de près le processus d'élaboration et de réalisation du spectacle AfroBrasil, conçu par le groupe de batucada Zalindê, en décembre 2008. A partir d'une approche ethnographique de la production artistique, j’ai essayé de comprendre le travail mené par les musiciennes qui ont participé à ce projet, à la fois à travers les activités quotidiennes, mais aussi à travers la nature collective des créations, les problèmes rencontrés, les solutions trouvées au fil du temps et les changements de programme qui ont eu lieu durant la préparation du spectacle.

Lié à la fois à un certain imaginaire brésilien et à la scène artistique actuelle, le groupe Zalindê fabrique des musiques nouvelles « d'inspiration brésilienne ", tout en préservant l'attachement à une idée de " tradition ". Cependant, plutôt que de me concentrer sur cette tension récurrente entre la " fidélité à la tradition » et la " création musicale originale », je propose de centrer l'analyse sur l'activité musicienne : comment fait-on de la musique brésilienne à Paris ? De cette façon, je m’intéresse surtout à la manière dont ces individus façonnent une musique dite brésilienne au sein de leur groupe et comment celui-ci s'inscrit dans un réseau de batucadas parisiennes, avec ses multiples personnages, instruments, influences et particularités.

L'anthropologue Denis Laborde, en résonance avec la " pensée par cas " proposée par Jean-Claude Passeron et Jacques Revel (2005), affirme qu'il faut " renoncer à la défense des catégories instituées pour examiner la façon dont elles sont instituées par nos pratiques" (Laborde 2010 : 7). Ce renversement de perspective impose de nouveaux défis à l'observation ethnographique : au lieu d'analyser les pratiques musicales à travers les catégories - comme par exemple la tradition, la world music, le chamanisme, etc. - nous allons observer comment ces pratiques font émerger ces catégories. Ce projet intellectuel réitère l'idée selon laquelle les actions humaines fabriquent le monde dans son énorme diversité, car « nous reconnaissons alors que ce qui est créé 
s'explique par le faire et non le faire par ce qui est créé » (Laborde 2010 : 10). Ainsi, plutôt que d'évaluer comment la musique produite par le groupe Zalindê a été fidèle à une hypothétique " tradition musicale brésilienne ", je cherche ici à comprendre comment les musiciennes construisent leur propre conception de la tradition : en quels termes et modalités est-elle exprimée, souhaitée, refusée, nécessaire ou efficace ? De cette façon, mon objectif, ici, n'est pas de créer des typologies ou des classifications, mais d'observer comment les actions des musiciennes de Zalindê font émerger " une musique traditionnelle brésilienne». Pourquoi l'attachement à une " tradition » est-elle importante ou utile pour les musiciennes de Zalindê ? Comment le spectacle AfroBrasil fabrique-t-il une musique brésilienne comme « traditionnelle » à Paris?

Il convient de rappeler, à la lumière des travaux de Gérard Lenclud, que le terme " tradition " est souvent employé automatiquement, sans que cet usage déclenche pour autant une réflexion plus approfondie sur ses significations. Cette notion est généralement associée à trois idées sous-jacentes: à une forme de conservation du passé dans le présent ; à une sélection des contenus culturellement importants et à un mode de transmission essentiellement oral. L'acception courante de ce terme renforcerait « un cadre de référence intellectuel constitué par un système d'oppositions binaires (tradition/changement, société traditionnelle/société moderne) dont la pertinence se révèle tout à fait problématique si l'on affecte à ces oppositions une valeur générique " (Lenclud 1987 : 3). De cette façon, l'usage de la notion de tradition peut opérer un " préjugé culturel » dissociant d'un côté les sociétés modernes, marquées par le changement et l'évolution, et de l'autre côté les sociétés traditionnelles, caractérisées par la circularité et par la conservation toujours inchangée des pratiques culturelles. Gérard Lenclud nous montre encore que la tradition serait un " point de vue que les hommes du présent développent sur ce qui les a précédés, une conduite en fonction des critères rigoureusement contemporains " (Lenclud $1987: 8$ ). Ainsi, c'est le moment présent qui façonne le passé, à travers une interprétation de ce qui est censé avoir été.

La question qui se pose ici n'est plus alors celle de la tradition, mais de la référence à des traditions. Nous entendons par là une référence qui nourrit une expérience quotidienne faite d'interactions humaines construites dans un espace hybride par excellence. Sous le signe de la tradition, une nouvelle 
musique est mise en œuvre par des identités complexes, développées à partir d'une pratique musicale déjà mondialisée, à savoir « un signifiant planétaire globalisé » (Amselle 2001 : 12). Les groupes de batucadas ont établi des stratégies et des pratiques originales d'appropriation, qui sont autant d'occasions de renouveler les sonorités et de former de nouveaux jeux d'identification par la musique.

\section{La scène des batucadas parisiennes}

Quelques jours après mon arrivée en France, j'ai réalisé qu'il existait un véritable monde autour de la musique brésilienne à Paris. Invitée, en tant que percussionniste, à rejoindre le groupe Zalindê, je me suis engagée dans la préparation d'une présentation de 12 minutes, spécialement conçue pour le $5^{\text {ème }}$ Concours de batucadas de Paris. Le concours a eu lieu le 22 mars 2008, au Cabaret Sauvage, lieu de spectacle situé dans le Parc de la Villette. Neuf groupes participaient à la compétition : Batuk'Addict, Batuk'Heroes, ENS'Batucada, Hanabi Sensei, Maracatu Oju Obá, Muleketu, Pernambucongo, Sambatuc et Zalindê. Etablies par les dirigeants des groupes, les règles du concours permettaient une grande liberté de création, en imposant seulement deux contraintes : la mise en place d'un répertoire "d'inspiration brésilienne " et l'utilisation d'instruments de percussion. Avant le début des présentations, je me suis promenée dans les loges, étonnée de retrouver à Paris une ambiance à la fois si familière et si étrangère. Musicienne et brésilienne moi-même, j’ai déjà maintes fois parcouru ces lieux très particuliers où les artistes se réunissent et s'échauffent, où les instruments s'entassent, les commentaires s'échangent sur les musiques et les musiciens, de nouvelles connaissances se font... Toujours dans l'attente impatiente d'enfin occuper la scène. J'ai pu, ici, retrouver des instruments, des costumes, des gestes, des chansons et des rythmes brésiliens inscrits dans un contexte qui m’était jusque-là complètement inédit, parler en français, avec de nouveaux accents, de nouvelles façons de faire et de vivre cette musique. J'étais alors en présence d'un réseau de professionnels et d'amateurs passionnés par la musique brésilienne percussive qui se consacraient à son apprentissage et à sa diffusion, qui revendiquaient des filiations avec tel ou tel groupe brésilien inspirateur et qui montraient avec aisance leurs choix artistiques et esthétiques. 
Autrement dit, un groupe de personnes en mouvement et en pleine expansion, qui élabore de nouvelles sonorités et formes de convivialité autour de la musique brésilienne.

De nouveaux espaces de convivialité et de sociabilité sont établis autour de ces groupes qui rassemblent des musiciens amateurs et professionnels, des français et des personnes d'origines diverses, réunis pour faire résonner les tambours brésiliens. Les répétitions et les représentations des batucadas sont des moments propices aux rencontres, au partage d'expériences, où se façonne une musique percussive forte et polyrythmique, mais où se tissent également des liens personnels et sociaux. Ainsi, si musicalement le mot batucada demeure imprécis et ouvert - car il désigne soit la musique produite par un groupe de percussions " d'inspiration brésilienne ", soit le groupe luimême sans donner davantage d'informations sur ses caractéristiques musicales ou sur sa formation instrumentale - , il nous apprend beaucoup sur un mode très particulier de production musicale. Bien que l'appellation « batucada " soit largement utilisée par les médias et les maisons de production et qu'elle soit adoptée par quelques groupes, l'existence de cette catégorie est largement questionnée par d'autres qui la refusent, affirmant que l'homogénéité des musiques et des pratiques que ce label nous laisse imaginer, n'existe pas vraiment.

Pratique musicale collective par excellence, festive et déambulatoire, la batucada porte l'imaginaire d'un Brésil carnavalesque, plein de joie, d'énergie et de sensualité. La danse et les chorégraphies exécutées par les musiciens sont une caractéristique fondamentale de ces représentations, souvent accompagnées d'une touche humoristique, irrévérente et presque théâtrale. « Des rythmes afro-brésiliens emmenés sur des chorégraphies épicées et enivrantes $^{2}$ ", pour citer quelques-unes des expressions utilisées par les groupes eux-mêmes, composent " une musique énergique et festive ${ }^{3}$ " dans une " performance chorégraphiée où rythme et danse ne font qu'un ${ }^{4}$ ". Art de rue également modulable pour la scène, le collectif de la batucada s'impose par sa puissance sonore et par un contact proche entre les musiciens et un public « transporté avec énergie et sensualité dans le ballet chaloupé de percussions

2 Site internet du groupe Zalindê, http://zalindesuccessst.canalblog.com/, consulté le 12 octobre 2008.

3 Site internet du groupe Zabumba, http://www.zabumba.org/, consulté le 12 octobre 2008.

4 Site internet du groupe Muleketu, http://seantahiri.free.fr/movie.html, consulté le 12 octobre 2008. 
mobiles ${ }^{5}$ ". Bref, le répertoire interprété par ces groupes serait " assaisonné à la brésilienne, avec ce qu'il faut de sensualité et d'énergie pour faire monter la température ambiante ${ }^{6}$ ", pour alors " faire danser et vibrer la foule sur des rythmes enflammés ${ }^{7}$ ".

Comme nous en avons parlé précédemment, malgré la diversité des groupes, le répertoire et la formation instrumentale qui fondent leur musique sont étroitement liés à trois manifestations culturelles brésiliennes clairement reconnaissables : la samba-reggae, l'école de samba et le maracatu. Remarquons aussi que les batucadas établissent différentes postures par rapport aux manifestations culturelles qui les inspirent. Celles-ci peuvent constituer une source d'inspiration très libre par un répertoire initial de rythmes et une formation instrumentale, ou un modèle à suivre le plus rigoureusement possible. Les liens de proximité entre les batucadas et ses groupes inspirateurs peuvent être affirmés de diverses manières : à travers une relation de filiation directe, par la réalisation de stages au Brésil ou par l'incorporation des habitudes et des façons de faire particulières. Voici des extraits qui, dévoilant leurs discours, nous montrent les termes de cette pratique.

La passion de la Samba a mené Sambatuc jusqu’à Rio où les percussionnistes se sont formés dans les meilleures écoles de Samba Carioca et ont défilé sur le Sambodrome tous les ans depuis l'an $2000 .^{8}$

Aquarela, de par son histoire, a une forte influence carioca. Influence qui se renforce par les liens d'amitié qui l'unissent à Portela. Comme toutes les écoles de samba, Aquarela a ses propres couleurs : orange et bleu. ${ }^{9}$

Pernambucongo est la filiation en France de l'école de percussions brésilienne fondée par Mestre Bernardino José, directeur musical de l'ensemble Maracatu

\section{Nação Pernambuco1o.}

5 Site internet du groupe Muleketu.

6 Site internet du groupe Zalindê.

7 Site internet du groupe Zabumba.

8 Site internet du groupe Sambatuc http://www.sambatuc.com/, consulté le 12 octobre 2008.

9 Portela est une des plus importantes écoles de samba brésiliennes. Site du groupe Aquarela http:// www.aquarela-paris.com, consulté le 10 février 2009.

10 Site internet du groupe Pernambucongo http://pernambucongo.fr/Index/Index.html, consulté le 10 février 2009. 
Le groupe Maracatu Nação Oju Obá vous invite à voyager à travers les rythmes du nord-est brésilien, à la découverte d'une culture riche en couleurs et en danse. (...) Oju Obá perpétue cette tradition, pour votre plus grand plaisir. ${ }^{11}$

A Paris et à Marseille, l'association Muleketu développe ses activités autour du samba-reggae : sa musique, sa danse, son patrimoine culturel. ${ }^{12}$

À l'inverse, d'autres batucadas adoptent plutôt la dimension musicale et festive des groupes de percussions brésiliens. Ils choisissent une formation instrumentale de base (fondée sur l'école de samba, le samba-reggae ou le maracatu comme nous l'avons vu précédemment) et s'approprient un répertoire de rythmes et de phrases " classiques " pour alors les mélanger librement à d'autres issus de diverses origines. A cet égard, Zalindê compose son répertoire en " mariant les styles et les couleurs au gré de ses envies: samba, merengue, ijexá, maracatu, hip-hop, raï, rythmes africains, samba-reggae... mettant en valeur tantôt la rythmique pure d'une influence, tantôt ses équivalences avec d'autres, privilégiant toujours la beauté des arrangements ainsi qu'une solide technique13 ". ENS'Batucada, par exemple, revendique une autonomie créative, un affranchissement vis-à-vis des standards rythmiques déjà établis et se définit plutôt comme un " collectif rythmique sur le modèle instrumental de la Batucada brésilienne » et non pas comme un héritier d'une quelconque tradition.

La plupart des batucadas concernées par cette étude s'organisent administrativement et économiquement autour d'une association culturelle, la loi 1901. La simplicité des formalités concernant sa constitution permet aux membres d'un groupe, ayant des projets artistiques, d'avoir rapidement une existence juridique, ce qui permet de gérer des ressources, réaliser des prestations, proposer des ateliers à toute la communauté parisienne, acheter des instruments, demander des subventions, etc. La structure organisationnelle d'une batucada repose donc sur le travail associatif, reparti entre différentes équipes qui sont responsables de la coordination des principales actions proposées par le groupe : les prestations et les ateliers.

Des ateliers de percussions brésiliennes sont proposés aux associations

\footnotetext{
11 Site internet du groupe Oju Oba http://afrocubop.nexenservices.com, consulté le 12 octobre 2008.

12 Site internet du groupe Muleketu.

13 Site internet du groupe Zalindê.
} 
durant toute l'année, destinés à un large public d'amateurs, débutants, musiciens confirmés, percussionnistes et batteurs. Généralement animés par le chef d'orchestre, ces ateliers constituent l'épine dorsale de la pratique musicale batucada puisque c'est à travers le renouvellement constant de ses membres que les groupes réussissent à diffuser leur musique et à former un collectif de personnes engagées à sa réalisation.

Centrée sur la pratique instrumentale, la pédagogie des batucadas met en valeur le caractère spontané et convivial de cette musique. Elle ne se démarque pas par un effort de théorisation mais par la tentative d'intégrer tous ces membres dans la création d'un collectif musicalement efficace. De cette façon, les ateliers sont orientés vers l'interprétation d'un répertoire de rythmes, de phrases rythmiques ou de morceaux déjà composés. Il est possible d'observer que ce résultat immédiat engendre un sentiment de satisfaction chez les élèves, car ils peuvent faire de la musique dès la première séance, sans avoir une pratique musicale préalable. Si la technique de jeu et la connaissance musicale nécessaires, afin de participer à une batucada, se révèlent minimales pour le débutant, ces tâches sont plus complexes au fur et à mesure de leur approfondissement. Bien que la facilité d'accès à cette pratique soit l'une de ses caractéristiques primordiales, cette étude entend montrer qu'elle ne dispense nullement d'un intense travail d'entrainement corporel et du développement de nouvelles compétences d'écoute de la part des participants.

Dans les situations de répétition, avant de faire de la musique, un rituel de préparation se déroule : il faut aller chercher les instruments, les accorder et les mettre en place dans le bon ordre, mettre la ceinture, la protection auditive et les genouillères, choisir la meilleure paire de baguettes disponible et attendre les premiers gestes du chef d'orchestre. Une dynamique d'apprentissage collectif s'instaure et la musique est transmise oralement à travers le trinôme observation-répétition-mémorisation. Les débutants apprennent sur le tas, en regardant les plus expérimentés, en intégrant les gestes par les gestes d'autrui, le tout dans une concentration maximale. En procédant sans l'aide de l'écriture musicale, la mémoire joue un rôle fondamental, car tous les musiciens doivent savoir tous les morceaux par cœur. Cela n'exclut pas d'éventuels enregistrements audio, utilisés comme aide-mémoire ou le recours à la notation musicale pour noter quelques idées. Pourtant, le processus de transmission demeure 
essentiellement oral car il n'est nullement besoin de maitriser le solfège pour participer à une batucada. A la fin des activités, les mouvements prennent le sens inverse, les instruments retournent au dépôt, les affaires personnelles sont rangées.

Jouer dans une batucada suppose un apprentissage du temps dans le sens le plus large du terme. C'est attendre le temps d'apprentissage de l'autre, répéter la même séquence rythmique jusqu’à la bonne exécution, agir dans le temps musical, autrement dit, insérer sa phrase rythmique dans une polyphonie rythmique très serrée. Se laisser imprégner par les rythmes et les mouvements, savoir réagir rapidement face aux difficultés de la situation de performance et bien répondre aux gestes du chef d'orchestre. La batucada implique un intense entrainement musical et corporel, la condition physique pour porter des instruments lourds et surtout la volonté de travailler en équipe.

Il faut encore préciser que les groupes de batucada se présentent, en général, en plein air, sous la forme d'animations déambulatoires et dans le cadre de fêtes de rue, de cortèges ou de carnavals. Autrement dit, des lieux ouverts dans lesquels la puissance sonore des instruments de percussions peut s'exprimer et où les musiciens peuvent exécuter leurs gestes et leurs chorégraphies en toute liberté. La musique batucada s'inscrit donc dans le paysage sonore de la rue, engendrant un "amalgame sonore» caractéristique de ces rencontres, fruit du mélange de bruits des tambours, de conversations, de voitures... Comme les autres groupes de batucada, Zalindê avait comme pratique musicale habituelle des présentations à ciel ouvert, régies par une grande convivialité, par la proximité avec le public, par une liberté de gestes et de déroulement. Pour réaliser un spectacle dans un théâtre, le groupe a mis en action un important changement de programme et a instauré de nouvelles pratiques et de nouvelles attentes pour " faire fonctionner " sa musique dans ce contexte jusqu'alors inédit. Force est de reconnaitre que le "format spectacle " implique un rituel très particulier, parfois très éloigné des situations de performance en plein air. Autrement dit, une pratique musicale qui a été initialement conçue pour être présentée lors d'une animation de rue et qui a été transposée dans la salle de concert - un espace qui demande des modes différents d'écoute, d'interaction et d'engagement corporel. 


\section{Zalindê}

Créé en 2002, Zalindê est un groupe de musiciennes amateures et professionnelles qui ont une passion en commun : la percussion brésilienne. C'est le fruit de la rencontre musicale de ses deux fondatrices : la percussionniste Roberta Paim et la chanteuse Chloé Deyme. Le nom du groupe a été élaboré à partir de la déformation en verlan du mot « lindeza ", qui signifie beauté et grâce en portugais. Première batucada féminine en France, d'après ses membres, Zalindê s'impose comme l'un des groupes les plus reconnus de la scène parisienne et a joué dans plusieurs villes d'Europe.

C'est pendant les répétitions du groupe $\mathrm{Muleketu}^{14}$, en 1999, que Roberta et Chloé se sont rencontrées et ont construit une solide amitié. Le projet de créer une formation exclusivement féminine au sein de ce groupe existait déjà, car Roberta, qui suivait une formation de chef d'orchestre, avait dirigé quelques concerts avec un petit ensemble de filles. Cependant, en 2001, une scission au sein du Muleketu a entrainé la sortie du groupe de Roberta et de Chloé, qui intégrèrent alors une nouvelle batucada nommée Vira-Lata. A cette époque Roberta, enceinte de sa fille Léa, s'est éloignée provisoirement de la pratique musicale pour y revenir quelques mois plus tard. Alors que le groupe Vira-Lata s'est rapidement dissout, Zalindê faisait ses premiers pas. Ultérieurement, les instruments et la structure organisationnelle de ViraLata ${ }^{15}$ ont été offerts à Roberta et Chloé, ce qui a rendu économiquement et matériellement possible la création de Zalindê. Elles se sont donc retrouvées toutes les deux dans un studio de répétition - accompagnées de la petit Léa " dans une poussette et avec des mini-bouchons dans les oreilles ${ }^{16}$ » - pour lancer les bases musicales et esthétiques qui guideront la formation de Zalindê.

Son répertoire de rythmes, sa formation instrumentale, ses compositions, ses chorégraphies et ses costumes ont été élaborés à partir de la matrice musicale et culturelle de la samba-reggae. Musicalement, il s'est largement inspiré du célèbre groupe bahianais Ilê Ayê, qui interprète un style particulier de samba-reggae, nommé « samba-afro ». Ilê Ayê a joué plusieurs

\footnotetext{
14 Le groupe Muleketu s'est formé à la suite d'une scission du premier groupe de samba-reggae à Paris, Batalá.

15 L'association qui accueille actuellement Zalindê est encore celle constituée à l'époque de Vira-Lata : ARAE, Association Autre Rythme, Autre Esprit.

16 Entretien avec Chloé Deyme, réalisé le 15 juillet 2009
} 
fois en Europe, notamment dans des festivals à Paris, dans lesquels Roberta et Chloé ont eu l'occasion de connaître et de partager la scène avec les musiciens bahianais. Quelques années plus tard, Marivaldo Paim, l'un des chefs d'orchestre du groupe Ilê Ayê, habitant désormais Paris, s'est marié avec Roberta. Il devient alors une présence assidue aux répétitions et aux concerts de Zalindê, nous proposant également des ateliers de samba-reggae et de candomblé. Des stages, réalisés à Salvador avec le groupe féminin de samba-reggae Didá, ont fortement contribué à la construction d'une esthétique propre à Zalindê ainsi qu'a l'élaboration de ses performances scéniques, comme l'affirme Roberta :

" Nous sommes très influencées par l'énergie, par la façon d'être des musiciennes de Didá. Les connaître nous a montré que c'est possible de jouer du tambour en jupe et en talon, maquillées. De danser de belles chorégraphies et d'être féminines sur scène, tout en étant fragiles et fortes en même temps. Ainsi, notre but est d'avoir une bonne musique, techniquement parfaite si possible, avec une belle esthétique, comme nous l'avons vu chez Didá, mais qui nous est propre forcément. Chacun a sa personnalité puisqu'elles sont au Brésil et nous en France. Ici, nous sommes moins afro et nous mettons l'accent sur le côté glamour, luxe $\mathrm{e}^{17}$."

Des arrangements sophistiqués et une palette rythmique variée composent le répertoire du groupe qui se fait aussi remarquer par les belles chorégraphies exécutées par ses musiciennes. Autour des rythmes identifiés comme brésiliens viennent s'agréger une multitude de rythmes issus d'autres traditions musicales et que les musiciennes identifient comme africains, cubains, caraibains, européens.

\section{De nouvelles demandes, un nouveau projet : AfroBrasil}

Le spectacle AfroBrasil a connu sa première et unique représentation le 10 décembre 2008, au Théâtre Douze à Paris. Ce moment marquait l'aboutissement d'un processus de création dont l'élaboration et la concrétisation ont demandé 10 mois de travail. Projet sur lequel vont converger des actions et des attentes, qui justifient les longues heures consacrées à sa réalisation,

17 Entretien avec Roberta Paim, réalisé le 2 août 2009. 
l'AfroBrasil signifie beaucoup pour ses créateurs : une articulation dans leur carrière professionnelle, une voie d'accès à des univers musicaux inattendus, un changement significatif dans leur façon de concevoir le travail collectif et la musique batucada.

Le projet prend naissance en mars 2008, à la suite du $5^{\text {ème }}$ Concours de batucadas de Paris, pour lequel le groupe a conçu une performance de $12 \mathrm{mi}-$ nutes, avec pour thème l'héritage africain dans la musique percussive brésilienne. Pour cette édition, les organisateurs du Concours ont proposé un changement significatif dans le dispositif de mise en scène des groupes. Au lieu de se présenter parmi le public, comme dans les éditions précédentes, les batucadas ont occupé la scène du Cabaret Sauvage. Un nouveau champ de possibilités techniques a été alors offert aux groupes : l'éclairage, la sonorisation des instruments, l'utilisation du chant, etc. Lauréat du Concours l'année précédente - fait qu'il a donné une propulsion importante à sa trajectoire professionnelle - Zalindê a alors décidé d'innover et de prendre des risques. Au lieu d'élaborer un morceau musical " conventionnel », Roberta et Chloé ont essayé de créer une performance mélangeant musique, danse et chant. Pour cela, le thème de l'influence de la culture africaine dans la construction de la musique brésilienne constituait le fil qui reliait la prolifération des petits tableaux dont la performance était composée. Nous parlons ici de culture au singulier : la « culture brésilienne » et la « culture africaine ", comme si ces deux expressions faisaient référence à des univers transparents, homogènes et bien délimités. Ainsi l'ambition était de raconter musicalement, certes d'une façon synthétique, l'arrivée des esclaves africains, le métissage culturel qui s'en suit et les pratiques musicales actuelles fortement reliées à cette présence noire au Brésil, dont la batucada serait l'un des exemples les plus remarquables. Mais cette fois-ci, Zalindê ne remportera que le deuxième prix du Concours. Cependant, l'expérience incite Roberta et Chloé à élaborer le projet AfroBrasil. Ainsi, l'expérience vécue à l'occasion du concours, où la scène fait son apparition, les amène à l'idée de la construction d'un récit musical.

Au cours d'une première réunion, le projet a été présenté au groupe et tous les membres de Zalindê y ont adhéré avec enthousiasme. Plusieurs questions ont été posées : comment mettre en place un projet si osé pour un groupe très hétérogène, formé par des musiciennes professionnelles et des amateures, qui ne possèdent pas forcément une formation musicale et 
qui ont une pratique musicale en général limitée à la batucada ? Comment trouver un financement et un espace capable d'accueillir un concert de percussion ? En quoi consiste exactement la réalisation d'un spectacle? Quels sont les enjeux de la construction de cet événement et les difficultés qu'elle apporte ? Quelles sources et ressources doivent être mobilisées ?

Roberta et Chloé nous ont exposé leurs idées initiales. Le projet consistait à mettre en place un spectacle d'une durée d'une heure et demie, en utilisant seulement des instruments percussifs et le chant. A l'intérieur du spectacle, quatre volets viendraient illustrer les étapes symboliques de la trajectoire de l'influence africaine sur la musique brésilienne : la musique africaine « traditionnelle »; le voyage vers le Brésil, les premiers contacts et la résistance culturelle africaine ; le " métissage " qui s'en suit et les manifestations culturelles produites ; et finalement, la dernière partie serait consacrée à la batucada, puissante synthèse des influences, typiquement brésilienne, mais qui aujourd'hui réaffirme sa vocation métisse et se diffuse globalement, en développant de nouvelles pratiques musicales et de nouvelles sociabilités, comme c'est le cas à Paris.

Pour cela, six personnes ont été chargées de la direction artistique du spectacle, choisies de par leurs compétences et leurs expériences professionnelles. Isabelle Guidon, percussionniste spécialisée en musique africaine, était la responsable des morceaux " africains ", dont une grande partie avait déjà été composée à l'occasion du concours de batucadas de 2008. Le volet brésilien nous était confié, à Lucia Campos et moi, à l'exception des rythmes et des chants de candomblé, appris dans un atelier animé par le percussionniste et chef d'orchestre du célèbre groupe brésilien Ilê Ayê, Marivaldo Paim. Les chants et arrangements vocaux étaient à la charge de Chloé Deyme. Roberta Paim a composé le morceau de batucada qui allait faire la synthèse du récit, clôturant le spectacle. En tant que comédienne, Kena Cuesta était la responsable de la mise en scène, cherchant une unité esthétique et conceptuelle capable de relier les quatre volets et de les présenter de façon cohérente au public.

Quelques prémisses ont guidé le travail de création artistique du spectacle dès sa conception. Pour Kena et Chloé, il existait une nette distinction entre un concert et un spectacle, le premier étant déjà ancré dans la pratique musicale de Zalindê et l'autre un défi à accomplir. Un spectacle ne signifiait pas un simple regroupement de morceaux musicaux, mais une unité dotée de 
sens et de cohérence interne. Pour y parvenir, il fallait jouer, danser et chanter, mettre en évidence l'aspect scénique et théâtral de la performance, ainsi que le « contenu " des morceaux, c'est-à-dire le message à diffuser à travers nos choix musicaux. Comme nous pourrons le remarquer plus loin, ces exigences plurielles feront monter considérablement la pression sur les musiciennes et le niveau de stress durant le processus de réalisation du spectacle. Après de longues discussions, il a été décidé que le spectacle serait articulé autour de 4 volets:

1 - « L’Afrique » : Passé lointain, les racines, ailleurs, temps mythique, commencement.

2 - « Le voyage » : La traversée des esclaves en bateau vers le Brésil.

3 - «Le métissage » : Passé plus proche, passé qui est encore présent aujourd'hui.

4 - « La batucada » : Aujourd'hui, ici, le temps présent, nous, notre identité en tant que groupe ; notre pratique musicale la plus familière, la samba-reggae.

Par conséquent, sans en avoir pleinement conscience, nous venions d'établir les lignes générales et les bases esthétiques qui inspireraient la construction du spectacle : une formation instrumentale percussive mariée au chant ; le maintien d'une idée de tradition et d'authenticité, mais également la quête de morceaux originaux et inédits ; la conception d'un spectacle à la fois scénique et musical ; un thème (l'héritage africain et le métissage musical) et un " scénario " à suivre minutieusement ${ }^{18}$.

Avec l'ambition de dresser une histoire musicale capable de relier un passé lointain au présent, le spectacle visait l'élaboration d'une narration en quelque sorte pédagogique. Il s'agissait d'un plan audacieux, qui se proposait de raconter, sous une forme musicale, le récit de l'arrivée des Africains au Brésil et le processus de métissage qui est au cœur de la formation de la société brésilienne - mise en abîme qui suppose la narration du mythe d'origine de cette musique elle-même.

Le dépliant distribué à l'occasion du spectacle montre une synthèse du travail mené et des conceptions esthétiques du groupe :

18 Après notre première représentation, nous avons réalisé que le scénario du spectacle n'était clairement compréhensible que pour les membres du groupe. Le public avait perçu le spectacle comme une succession de petits tableaux musicaux, pas forcément reliés entre eux. 
Un spectacle vivant qui tisse le lien entre les percussions de l'Afrique et du Brésil ! Les 18 musiciennes de la troupe Zalindê mettent en lumière les premiers pas de la culture africaine au Brésil, et se jouent des comparaisons et similitudes encore possibles aujourd'hui entre les deux univers. Des djembés du Mali aux tambours de Minas Gerais, il y a un monde... pas si sûr ! Au travers des percussions, du chant et de la danse, ces 18 artistes dévoilent un répertoire riche d'arrangements novateurs, de compositions originales et de morceaux traditionnels. Un spectacle qui exalte la force de l'évolution et l'héritage inaliénable de la culture afro-brésilienne. Venant d'horizons différents, elles sont originaires du Brésil, du Sénégal, du Bénin, mais aussi de France métropolitaine, des Antilles Françaises, du Maroc, de Colombie, du Vietnam et du Japon. Ce sont donc autant de cultures et sensibilités différentes, qui se sont penchées sur le phénomène de métissage musical que l'on peut observer au Brésil, pour nous offrir aujourd'hui « AFROBRASIL ».

Le format « spectacle » imposera-t-il alors un fort changement dans les pratiques habituelles du groupe ? Quelles seront les implications et les conséquences de ce choix ? Curieusement, les pratiques et les manières de faire du groupe n'ont pas subi une grande transformation car le travail quotidien de construction du spectacle consistait à composer, répéter, mémoriser, apprendre par cœur les morceaux et les chorégraphies, des tâches déjà très familières aux membres de Zalindê. Pourtant, ce nouveau projet avait quelques spécificités qui ont entrainé des transformations importantes, liées particulièrement au changement d'espace de la présentation, à la multiplication des directrices, à la superposition de tâches à accomplir et à l'introduction d'un nouveau répertoire de pièces et de rythmes.

\section{La tradition conjuguée au présent}

Nos discussions préliminaires ont révélé des points de vue fortement divergents sur le répertoire à mettre en place et sur la façon de le construire. Devons-nous exécuter des morceaux inédits ou interpréter des rythmes et des chansons " traditionnels " ? Ces deux répertoires peuvent-ils coexister dans un même spectacle ? D’un côté, Isabelle désirait fabriquer le volet africain le plus « traditionnel " possible, en utilisant une formation instrumentale 
constituée de trois djembés ${ }^{19}$, trois dunduns ${ }^{20}$ et des diabaras ${ }^{21}$. De l'autre, Chloé affirmait catégoriquement la nécessité de développer de nouvelles phrases rythmiques et de nouvelles compositions où le chant serait présent, y compris en langue française. Curieusement, cette discussion était plutôt une affaire entre les « experts » du groupe et ne mobilisait guère l'attention des autres musiciennes, qui pour la plupart partageaient l'opinion de ne pas établir de bornes trop rigoureuses entre la musique « traditionnelle » et celle dite «inédite ". Nos débats se dressaient autour d'une idée de la tradition : j’ai pu maintes fois remarquer que ces idées n'ont pas de correspondance entre leurs versions brésiliennes et parisiennes. Autrement dit, une pratique qui peut être rangée sous l'appellation de « traditionnelle » à Paris ne l'est pas forcément au Brésil, comme par exemple la samba-reggae ${ }^{22}$.

Voyons de plus près la construction musicale de deux morceaux qui composent le volet " brésilien " du spectacle et dont le maracatu est le rythme de base. Dans le premier, A bandeira é brasileira ${ }^{23}$, nous avons essayé de suivre l'architecture sonore du maracatu telle que nous l'avons apprise à Recife, ou lors des ateliers avec des musiciens issus de cette tradition. Cela signifie, non seulement une formation instrumentale particulière (composée de plu-

19 D'origine africaine, le djembé est un instrument de percussion en forme de calice. Son corps est composé par un fût de bois et une peau de chèvre tendue grâce à un système de cordages. On en joue à mains nues.

20 Le dundun est un instrument de percussion africaine sculpté à la main dans une seule pièce de bois creux, de forme cylindrique. Cache extrémité de l'instrument porte une peau animale et sur son corps est attaché une cloche de métal. Il est généralement joué dans ensemble composée de trois dunduns accordés différemment. Sa technique de jeu : les musiciens frappent la peau de l'animale à l'aide d'une grosse baguette et la cloche avec l'autre main, avec une tige de fer ou un anneau.

21 Instrument de percussion aussi d'origine africaine, le diabara est une sorte de hochet construit avec une calebasse et garni d'un filet de coquillages. Le musicien tient la calebasse d'une main pendant que l'autre main fait bouger l'extrémité du filet.

22 Voici un autre exemple de différentes logiques d'attribution du caractère " traditionnel " aux objets et aux pratiques. Le Diplôme d'études en Musique (DEM) delivré par les Conservatoires à Rayonnement Régional ou Départemental français propose actuellement une formation en musique traditionnelle. Parmi les mentions possibles figure celle intitulée " percussions traditionnelles brésiliennes ", en l'occurrence dirigée par le professeur et percussionniste Paul Mindy. Julie Ho, membre du groupe Zalindê et qui actuellement suit cette formation, nous raconte que l'apprentissage d'une vaste gamme d'instruments est offert aux élèves, tels que le pandeiro, les atabaques, le berimbau, les alfaias, la caisse claire, le tambourim, etc. Le contenu pédagogique de la formation regroupe des rythmes comme l'école de samba, le choro, la samba, le maracatu et la capoeira, pratiques musicales qui seraient difficilement rangées ensemble au Brésil sous l'étiquette « musique traditionnelle ».

23 Loa attribuée à la Nação de Maracatu Leão Coroado, l'un des groupes les plus anciens de Pernambouc. 
sieurs alfaias, caisses-claires, ganzás, abês et de gonguê) mais également une gestualité spécifique, une organisation spatiale sous forme de cortège et un répertoire de variations rythmiques possibles. Le morceau suivant, Maracatu Zalindê, maintient la même instrumentation mais présente un arrangement plus libre des contraintes stylistiques, par rapport au maracatu considéré comme " traditionnel ". Ainsi nous avons créé une introduction composée de petits commentaires rythmiques, joués séparément par les instruments qui forment une mélodie de timbres en étroit rapport avec les paroles de la chanson. De plus, le rythme exécuté par la caisse-claire a subi un changement dans la deuxième partie du morceau, c'est-à-dire qu'au lieu de jouer le rythme traditionnel, l'accent fort est décalé vers le deuxième et le quatrième temps de la mesure, à la manière des rythmes funk et rock. À ce moment exact, les musiciennes qui ne participaient pas au tableau rentrent sur scène en dansant librement. À la fin du Maracatu Zalindê, elles quittent la scène encore une fois, dans une explosion collective d'énergie. Nous pouvons observer, ici, que l'intitulé " traditionnel » ajouté au premier morceau n’a pas été employé d'une façon arbitraire. Certains critères ont guidé cette distinction et ont été discutés collectivement puis acceptés tacitement par les musiciennes du groupe, car ils pouvaient être justifiés et expliqués à posteriori. Cependant, l'attribution d'une " traditionnalité " ne pourrait pas être considérée comme intrinsèque aux pratiques musicales ou répertoires concernés, car elle s'applique différemment selon les contextes et moments socio-historiques.

Pendant l'élaboration du spectacle, rien n'est figé. Le sens du mot « tradition " est façonné à travers les choix et les découpages réalisés par le groupe, dans un processus continu de construction et de remise en question de son propre répertoire. Par exemple, les situations concrètes nous imposent, à la fois, des contraintes et de possibles solutions. Que faire s'il manque un instrument traditionnel, s'il manque la technique nécessaire pour en jouer, ou l'expérience préalable pour bien exécuter un rythme ? Il a constamment fallu négocier des solutions ; construire notre spectacle à partir de nos ressources et nos compétences ; effectuer des changements de programme ; ajuster les arrangements et les compositions à la réalité musicale et financière du groupe.

J'ai pu observer qu'à l'intérieur du spectacle AfroBrasil, le critère qui établit la différence entre un " morceau traditionnel » et un " morceau non-traditionnel » réside dans le maintient ou la transformation d'un ou plusieurs des aspects de la pratique musicale « traditionnelle » inspiratrice. Suivre la 
tradition, c'est d'abord respecter des contraintes : une rythmique, un répertoire de chansons, le déroulement d'une dramaturgie particulière, une formation instrumentale, une gestualité, des costumes, etc. Des contraintes établies, d'ailleurs, par des " passeurs " entre la tradition et le groupe : en l'occurrence, Lúcia, Isabelle et moi-même. Si, d’une part, notre expérience professionnelle en tant que percussionnistes et notre connaissance musicale se révélaient fondamentales pour la construction du spectacle, d'autre part nous ne revendiquons pas l'appartenance à une tradition musicale spécifique, ni la défense de celle-ci. C'est une relation de confiance et de mise en œuvre collective de compétences qui s'est établie à l'intérieur du groupe et qui nous a investies de ce pouvoir décisionnel : car nous ne serions jamais considérées au Brésil ou en Afrique comme des musiciennes garantes d'une quelconque tradition.

En résumé, une constante tension créative entre " innovation » et " tradition " a été présente pendant les travaux de composition et d'arrangement des morceaux. En mélangeant dans son répertoire l'interprétation de morceaux « traditionnels » et de nouvelles pièces jouées « de façon traditionnelle ", Zalindê pourrait toujours revendiquer son identité, l'autorité de son répertoire et de ses compositions. Autrement dit, il était possible d'assurer la propriété artistique de sa création musicale, sans pour autant se détacher d'un discours fondé sur la musique traditionnelle. On aura bien compris combien cette ambiguïté (tradition / innovation) a été nécessaire au groupe pour préserver, à la fois, l'ancrage dans une tradition musicale - qu'elle soit africaine ou brésilienne - et une identité propre en tant que groupe de percussion, inscrit dans le temps présent et dans un contexte particulier. Cette ambiguïté présente l'avantage de laisser ouvert le champ des choix et des sources musicales qui peuvent être mis en œuvre dans une création. De la sorte, les musiciennes peuvent adopter une interprétation la plus fidèle possible d'une musique, avec ses instruments, sa grammaire, ses techniques de jeux et sa gestualité caractéristique, ou la transformer au gré de leurs désirs et de leurs besoins de création. De même, les formules rythmiques inspirent la naissance de chansons inédites et de nouvelles compositions voient le jour à partir de collages de rythmes " traditionnels ", de " phrases originales » dans une élaboration qui revendique un auteur et une façon de faire particulière. Ici, je pense encore une fois à la notion de tradition telle qu'elle est conçue par Gérard Lenclud : « L'utilité, en général, d'une tradition, est de fournir au 
présent une caution pour ce qu'elle est : en l'énonçant, une culture justifie d'une certaine manière son état contemporain " (Lenclud : 1987, 9).

\section{Pratique musicale et recherche ethnographique}

Si les difficultés imposées au chercheur, qui étudie un monde artistique auquel il participe, sont déjà significatives, ma situation personnelle en tant que membre du groupe et directrice musicale du spectacle s'est avérée particulièrement délicate. Force est de reconnaître qu'étant brésilienne, percussionniste et ethnomusicologue, j'étais, pour les autres musiciennes du groupe, trop chargée de responsabilité et d'autorité. Au-delà d'un regard de l'intérieur, cela supposait un regard issu du centre de pouvoir sur lequel reposaient les décisions musicales et la gestion concrète des répétitions, des conflits et des problèmes surgissant tout au long de notre travail collectif. Mon engagement, en tant que directrice musicale, m'a poussée à donner une forme, une consistance et un aboutissement au spectacle, à prendre des décisions, à changer les places et les rôles des autres membres du groupe.

La décision de prendre le spectacle - jusque-là restreint à ma pratique musicale - comme objet d'étude a été prise lors de l'intensification du travail de création du morceau final de batucada « Machine de Guerre 2 » et à l'occasion de nos premières répétitions générales, quelques semaines avant la première. Il fallait alors mettre en place une stratégie capable, à la fois, de me permettre le passage du rôle de musicienne à celui d'observatrice et d'organiser une base de données susceptible d'être analysée a posteriori. Mais il fallait aussi organiser l'expérience vécue durant 10 mois pour rendre compte de l'intégralité du processus d'élaboration du spectacle dès ses premiers pas. Les musiciennes du groupe ont bien reçu ma proposition d'étudier la fabrication du spectacle. J’ai pu enregistrer nos répétitions et nos réunions, réaliser des entretiens avec les musiciennes et quelques personnes du public.

Howard Becker et Robert Faulkner, tous deux à la fois chercheurs et musiciens, ayant mené plusieurs enquêtes sur la pratique musicale des jazzistes, remarquent que cette proximité entre l'objet d'étude et le chercheur peut constituer une aide ou une limite. La connaissance préalable de la grammaire musicale et du vocabulaire utilisé par les personnes interrogées facilite l'approche, car quelqu'un d'étranger à ces langages peut mettre des mois à saisir leur dynamique. Cependant, il faut prendre garde à certains pièges tels que « 
l'exploration mutuelle ou l'explicitation de savoirs tacite ». Le danger majeur serait d'élaborer " des questions de telle sorte que les enquêtés comprennent facilement » et répondent exactement ce que le chercheur veut entendre (Becker et Faulkner : 2008).

Effectivement, la réalisation des entretiens avec mes collègues musiciennes a été un moment particulièrement délicat de mon enquête. D’abord, il fallait dépasser un certain artificialisme de la situation et créer une ambiance proche de celle de nos conversations quotidiennes. Certaines questions étaient impossibles à poser, elles déclenchaient le rire, ou des réponses comme celle-ci : comme tu le sais..., ou comme tu le disais lors des répétitions... J'avais l'impression de retrouver ce que je savais déjà, de repérer mon discours à l'intérieur du discours des autres musiciennes. Bien évidemment, après des mois d'intenses discussions autour des enjeux du spectacle, comme l'appropriation de la musique brésilienne en France, les approches possibles de la musique traditionnelle, les tensions entre la pratique " des amateurs" et celle " des professionnels » dans le milieu des batucadas, il était normal qu'il existe un discours qui soit en quelque sorte partagé, plus ou moins consensuel. J'ai pu donc remarquer que grâce à certaines certitudes partagées entre moi et les musiciennes du groupe, certains aspects de nos pratiques n’ont jamais été remis en question. Cela m’a empêchée de poser des questions très simples, comme, par exemple : « Quelle musique jouez-vous ? Comment définissez-vous la musique interprétée par le groupe ? " Bien évidemment, je n'ai pas posé ces questions parce que la réponse me semblait par trop évidente. En outre, je connaissais déjà la réponse. Dans ce cas précis, la façon conventionnelle de mener des entretiens ne s'avérait pas très efficace. Il fallait donc développer une autre stratégie d'approche, d'entretiens et d'observations, tout en étant très attentive à ne pas tomber dans ces vastes implicites, dans ces idées toutes faites, couronnés d'un silence de conviction partagée.

En regardant rétrospectivement mon engagement dans le milieu des batucadas, il s'avère que mon expérience au Brésil a eu une influence directe sur ce que Daniel Cefaï appelle « les savoir-faire qui comprennent l'accès au terrain (se faire accepter, gagner la confiance, trouver sa place, savoir en sortir...) " (Cefaï 2010 : 7). Un exemple : être percussionniste m’a permis d'être très vite acceptée au sein de Zalindê. J'ai pu accéder directement au groupe une semaine avant le $5{ }^{\text {ème }}$ Concours de batucadas de Paris, un moment particulièrement tendu et très intéressant. Mais cette prise de position m’a aussi 
plongée dans des relations tantôt d'affinité tantôt d'animosité envers les autres groupes. Le milieu des batucadas parisiennes est très compétitif et critique. Intégrer Zalindê impliquait une communication difficile avec d'autres groupes « rivaux ». Je ne comprenais pas l'indifférence et le mépris manifestés par certains chefs d'orchestre quand je leur disais que j'avais intégré Zalindê. C'était comme interroger un amateur de foot en portant le maillot de l'équipe adverse.

Mais, concrètement, comment mon expérience musicale et mon implication directe auprès de groupes de batucadas pourraient-elles faire avancer la connaissance de cette musique ? En d'autres termes, en quelle mesure le double regard de musicienne et d'anthropologue pourrait-il m'aider à décrire et à analyser ces pratiques musicales ? Premièrement, la connaissance du répertoire mis en place par le groupe rendait plus facile l'identification des sources sonores employées dans une création musicale (phrases, rythmes, chansons, instrumentations, etc.). Ainsi, après le repérage de certaines de ces sources musicales, il m'a été possible d'observer les façons selon lesquelles les compositrices les manipulaient, les transformaient et les articulaient dans de nouveaux contextes, en créant de nouvelles possibilités sonores, ainsi que des significations propres au groupe. Ce moyen a été très utile dans l'analyse du dernier morceau du spectacle, Machine de Guerre, dans lequel la batucada proprement dite occupe la scène. Cette pièce, composée par une succession de tableaux rythmiques qui s'inspirent des rythmes africains et brésiliens présentés durant le spectacle, m'a permis d'observer la transposition de rythmes de candomblé (généralement joués sur un ensemble de trois atabaques et une cloche) vers un groupe de 18 musiciennes munies d'instruments de sambareggae $^{24}$. En outre, j’ai pu également accompagner l'élaboration des costumes et des chorégraphies qui se rapportaient à l'univers mythique et gestuel des orixás concernés. Bien que ces références au candomblé soient difficilement repérables par un public qui n’est pas habitué à cet univers culturel ou qu'elles ne soient plus clairement reconnaissables à l'intérieur du morceau, la jonction de la danse et de la musique apportait une signification particulière

24 La formation instrumentale de base du groupe compte 4 types de tambours graves accordés différemment, nommés généralement " surdos " (marque 1, marque 2, double et coupe, le premier étant le plus grave et le dernier le plus aigu) ; les caisses-claires ; les petits instruments tels que le ganzá et les cloches; et finalement le repinique. Ces instruments possèdent une ample tessiture sonore, des plus graves, comme les marques, aux plus aigus, tel que le repinique. 
aux membres du groupe. Je cite ici un exemple : l'interprétation du rythme d’ijexá impliquait une chorégraphie et une intention précises, proches de celles attribuées à l'orixá Oxum.

Deuxièmement, j’ai pu aborder des questions difficiles liées à l'interprétation - mais qui sont fondamentales pour les musiciens, comme le " swing " par exemple - tout en essayant de décrire comment les pratiques, l'usage des instruments, la gestualité des musiciens et l'espace de répétition changent la sonorité d'une musique qui se déplace géographiquement. Autrement dit, observer et décrire très concrètement les façons dont se construisent musicalement les ambiances, les saveurs et les couleurs locales d'une world music.

\section{Conclusion}

Cette recherche a été une tentative de saisir les mécanismes de production et d'appropriation qui sont à l'origine de la création musicale fondée sur l'expérience parisienne des batucadas, à la fois mondialisée et singulière. En suivant de près le processus d'élaboration du spectacle AfroBrasil, nous avons dévoilé le façonnement collectif d'une musique brésilienne sur le sol français, en décrivant ses particularités et ses caractéristiques. Cela nous a montré qu'un processus de globalisation économique et culturelle, responsable de la diffusion mondiale de la musique batucada, ne s'accompagne pas nécessairement d'une standardisation des formes culturelles instituées par les pratiques musicales dites « inspiratrices ". Même si, dans ses grandes lignes, la musique produite par Zalindê ressemble à d'autres également inspirées de la matrice culturelle de la samba-reggae brésilienne, elle s'en différencie énormément par la façon dont elle a été conçue, ses sources et ses ressources, ses accents et sa sonorité. Ainsi, une dynamique active et originale est mise en œuvre dans les interactions sociales et artistiques produites à l'intérieur de cette rencontre entre musiciens brésiliens immigrés et francophones, musiciens " du monde », qui puisent dans cette référence mythique des traditions musicales brésiliennes idéalisées, en faisant de celles-ci un moteur de leurs propres créations musiciennes.

Pour conclure, j'aimerais parler très brièvement du dernier volet $\mathrm{du}$ spectacle, " la batucada ", dans lequel nous avons joué une pièce de trente minutes de durée, nommée Machine de Guerre 2008. En réalisant en quelque sorte une relecture de soi-même, la Machine de Guerre 2008 synthétise le 
programme musical de l'AfroBrasil, parcourant les rythmes africains, (joués dans le premier volet du spectacle) puis ceux dits brésiliens comme le candomblé, le congado et le maracatu (interprétés lors des seconds et troisièmes tableaux) pour enfin arriver à la samba-afro du groupe inspirateur Ilê Ayê. En opérant une mise en abîme qui met en relief le processus de création du spectacle lui-même, le groupe utilise les expériences vécues et les connaissances acquises pendant la période d'élaboration pour alors les transposer vers sa propre architecture musicale, mettant en scène une mosaïque musicale qui fonctionne comme la synthèse emblématique de cette aventure historiquemusicale dans un langage beaucoup plus familier : la samba-reggae.

Opérant le transfert d'une musique déambulatoire vers la scène du Théâtre Douze à Paris, les musiciennes ont dû faire face à plusieurs défis : de multiples contraintes techniques, bien sûr, mais également l'ouverture d'un nouveau champ de possibilités musicales et artistiques. Déplacements, transferts, influences, emprunts, héritages : AfroBrasil dresse un récit de déplacements de musiques et de cultures qui donnent naissance à de nouvelles musiques et à de nouveaux déplacements, dans une volonté explicite de fonder son propre mythe d'origine.

\section{Références bibliographiques}

AMSELLE, Jean-Loup. 2001. Branchement: anthropologie de l'universalité des cultures. Paris: Flammarion.

BECKER, Howard. 1988. Les mondes de l'art. Paris: Flammarion.

BECKER, Howard; FAULKNER, Robert. 2008. "Studying something you are part of: the view from the bandstand". Ethnologie Française, 2008(1): 15-21. BECKER, Howard. 2005. "Le répertoire du Jazz". In: Sociologie de l'art. Paris: L'Harmattan. pp. 15-24.

CEFAÏ, Daniel. 2010. L'engagement ethnographique. Paris: Editions EHESS. FOUCAULT, Michel. 1966. Les mots et les choses. Une archéologie des sciences humaines. Paris: Editions Gallimard.

FOUCAULT, Michel. 1969. L'archéologie du savoir. Paris: Editions Gallimard. HENNION, Antoine. 2007. La passion musicale: une sociologie de la médiation. Paris: Métailié.

LABORDE, Denis. 2005. La mémoire et l'instant: les improvisations chantées du bertsulari basque. Donostia: Elkar. 
LABORDE, Denis. 2010. Pour une science indisciplinée de la musique. [Texte en cours de publication].

LACOUR, Philippe. "Penser par cas, ou comment remettre les sciences sociales à l'endroit”. Article consulté le 23 mars 2009 sur le site http:/| espacestemps.net/

LENCLUD, Gérard. 1987. “La tradition n'est plus ce qu'elle était... Sur la notion de 'tradition' et de 'société traditionnelle' en ethnologie”. Terrain, 9: 110-123.

LENCLUD, Gérard. 2009. "Les cultures humaine et le bateau de Thésée : Le problème de l'identité des cultures dans le temps”. In: D. Laborde (org.), Désirs d'histoire: politique, mémoire et identité. Paris: L’Harmattan. pp. 221-248

LENCLUD, Gérard. “Identité et identités”. Article consulté le 23 mars 2009 sur le site http:/|www.cairn.info/

LENCLUD, Gérard. 1994. “Qu'est-ce que la tradition?”. In: M. Détienne (org.). Transcrire les mythologies. Paris: Albin Michel. pp. 25-44.

PASSERON, Jean-Claude; REVEL, Jacques. 2005. Penser par cas. Paris: Editions de l'EHESS, coll. Enquête.

\section{BIOGRAPHIE}

Doctorante en ethnomusicologie à l'EHESS sous la direction de Denis Laborde, Emilia Chamone a soutenu son mémoire de Master II dans la même institution. Percussionniste, arrangeuse et professeur de musique, Emília Chamone est diplômée en percussion à l'Université Fédérale de Minas Gerais, où elle a également réalisé un master II sous la mention éducation musicale. En tant que percussionniste elle a joué sur de nombreuses scènes à travers le Brésil et en Europe pour accompagner des artistes tels que Mariana de Morais, Kery James, Regina Spósito, Chico Lobo, Nelson Sargento, Vander Lee, Cirque National Alexis Gruss, Coral das Lavadeiras de Almenara, groupe Zalindê, Sarandeiros, Copo Lagoinha, Bantuquerê e Prucututrá, entre outros.

www.myspace.com/emiliachamone 